\section{$C_{\text {cituris }}$}

Department of Diagnostic Radiology, Mayo Clinic, Jacksonville, Florida, USA ${ }^{2}$ Department of Cardiovascular Diseases, Mayo Clinic, Jacksonville, Florida, USA

\section{Correspondence to}

Dr Chanel M Wood, Department of Diagnostic Radiology, Mayo Clinic, Jacksonville, FL 32224 USA; wood.chanel@mayo.edu

Received 18 March 2018 Revised 5 July 2018 Accepted 9 July 2018 Published Online First 24 July 2018

\title{
Systemic-to-pulmonary arterial connections simulating pulmonary emboli in a patient with Tetralogy of Fallot
}

\author{
Chanel M Wood, ${ }^{1}$ Christopher J McLeod, ${ }^{2}$ Pragnesh P Parikh, ${ }^{2}$ Carlos A Rojas ${ }^{1}$
}

\section{CLINICAL SCENARIO}

A middle-aged patient with a history of Tetralogy of Fallot repair (status post modified Blalock-Taussig shunt, right ventricular outlet tract reconstruction and ventricular septal defect repair, with more recent pulmonary artery patch annuloplasty with dilation and stenting, pulmonary valve replacement and aortic valve and root replacement) and symptomatic atrial tachycardia on coumadin therapy presented to an outside institution with dyspnoea and recurrent atrial fibrillation. The patient underwent CT pulmonary angiogram (CTPA), which demonstrated multiple unopacified areas in the bilateral segmental pulmonary artery branches; however, international normalised ratio was supratherapeutic at 4.1. The patient was transferred to our facility for suspected pulmonary emboli (PE). On assessment, the patient was noted to be in atrial fibrillation without evidence of hypoxia or respiratory compromise. In the context of adequate anticoagulation and a lack of clinical correlation, a repeat CTPA with pulmonary artery bolus tracking and imaging acquisition at 100 Hounsfield Units was performed which again demonstrated unopacified segments in the bilateral pulmonary artery branches (figure 1A). In addition to this standard CTPA protocol, an additional $28 \mathrm{~s}$ delayed acquisition targeted at predominantly systemic arterial phase opacification was performed. This additional sequence demonstrated contrast filling the previously unopacified segmental branches (figure 1B), excluding PE and consistent with an alternative diagnosis of systemic-to-pulmonary artery communications. Furthermore, a tangle of systemically opacified vessels was identified above the left atrium with supply from the sinoatrial nodal and bronchial arteries. Multiple connections between this tangle and the bilateral, proximal pulmonary artery circulation resulted in unopacified pulmonary segmental branches in the pulmonary phase imaging. An example of one of these systemic-to-pulmonary arterial branch connections is demonstrated in figure 2 .

\section{DISCUSSION}

Systemic-to-pulmonary artery communications, or major aortopulmonary collateral arteries (MAPCAs), are systemic arteries that supply the lungs in the setting of an underdeveloped pulmonary circulation (more frequently in the setting of pulmonary atresia) and are identified in up to $13 \%$ of patients with Tetralogy of Fallot. ${ }^{1}$ The most common sources of these anomalies-the
D Check for updates

(c) Author(s) (or their employer(s)) 2018. No commercial re-use. See rights and permissions. Published by BMJ.

To cite: Wood CM McLeod CJ, Parikh PP, et al.

Thorax 2018:73:1091-1092.

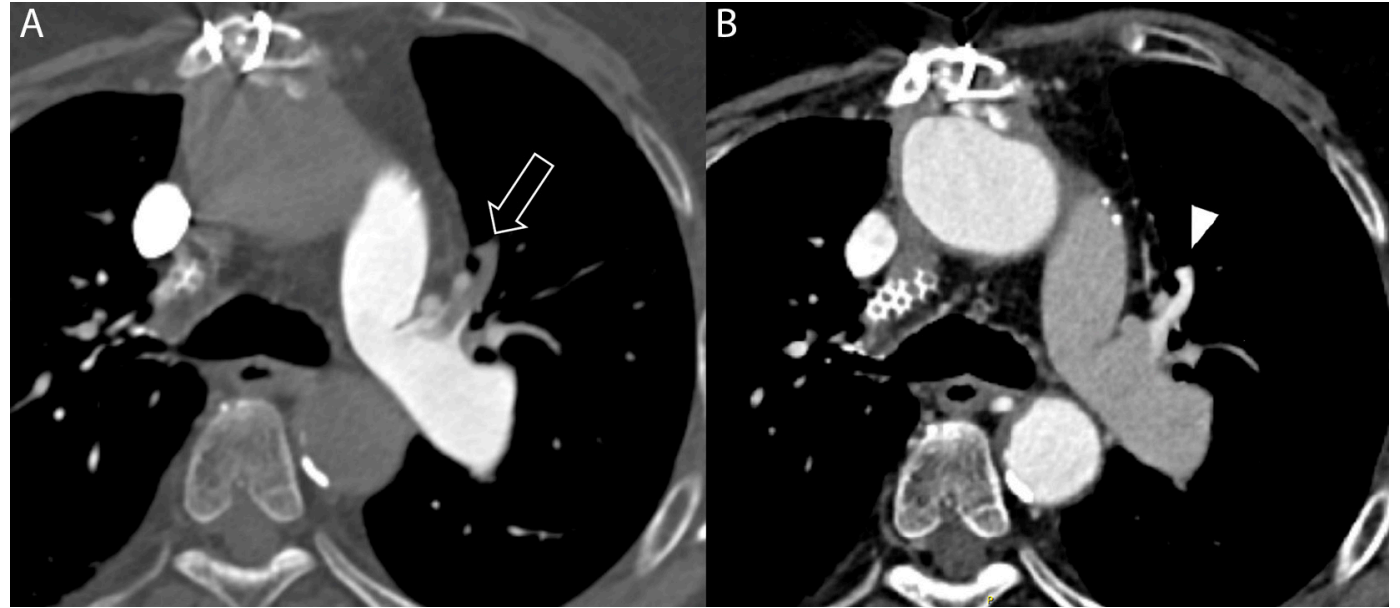

Figure 1 (A) Axial CT image of the chest after administration of contrast during the pulmonary arterial phase demonstrates an unopacified left upper lobe segmental pulmonary artery branch, identified by black arrow.(B) Axial CT image of the chest after administration of contrast during systemic arterial phase imaging (acquired at $28 \mathrm{~s}$ delay) demonstrates late opacification of previously unopacified segmental branch, identified by white arrowhead, compatible with MAPCA-to-pulmonary artery connection. MAPCA, major aortopulmonary collateral artery. 


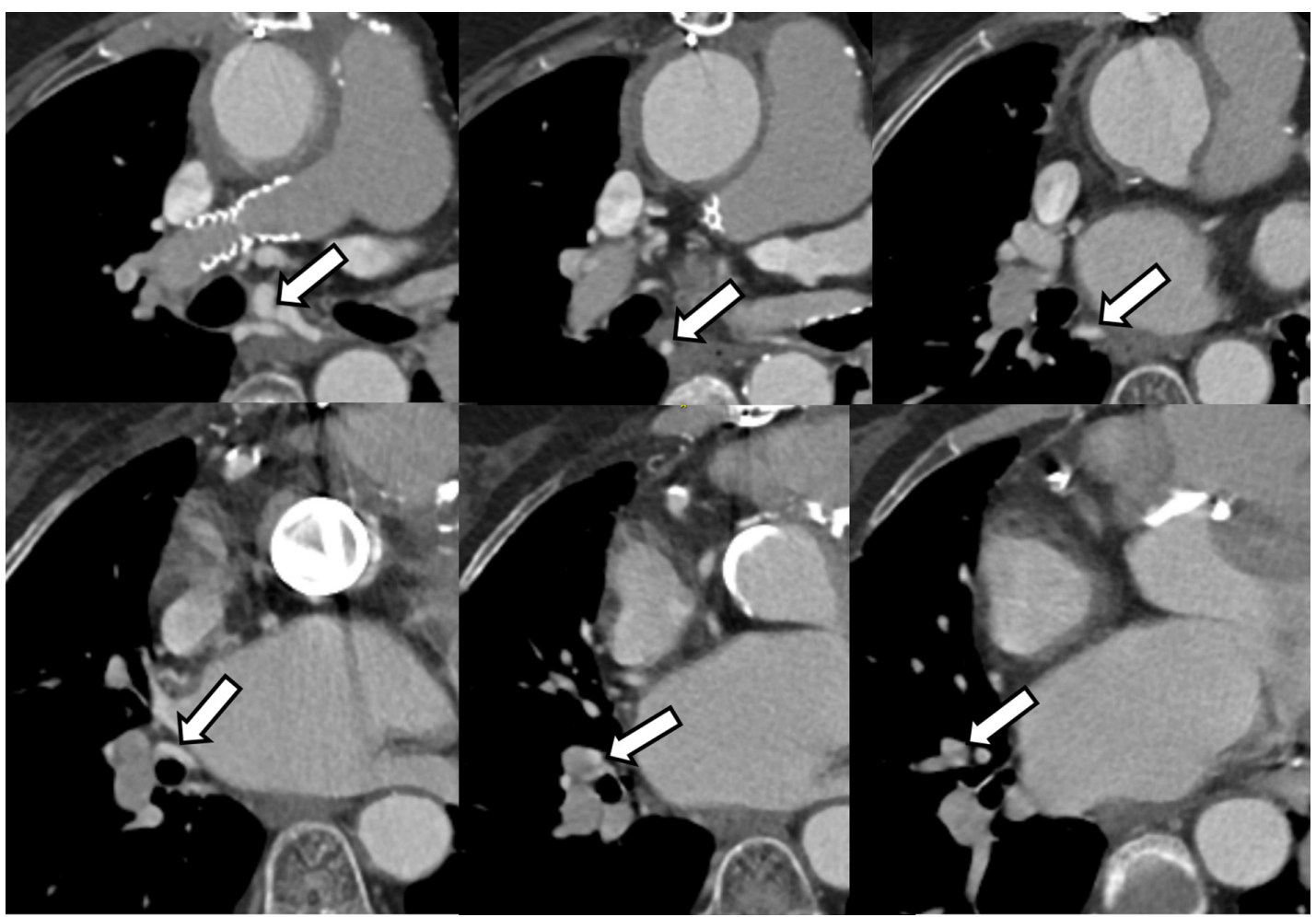

Figure 2 Axial CT images of the chest after administration of contrast (systemic arterial phase) demonstrate MAPCA (white arrows) extending into the right hilar region and connecting distally to the anterior segmental branch of the right lower lobe pulmonary artery. MAPCA, major aortopulmonary collateral artery.

descending aorta and subclavian arteries-are similar to those of bronchial arteries, supporting the hypothesis that they share a common developmental origin. ${ }^{2}$ The MAPCA tangle described above is a rare anomaly with dual coronary and bronchial sources and an opacification pattern consistent with systemic arteries. Using delayed imaging, PE was ruled out and this patient was spared continued workup and treatment; to the authors' knowledge, this is the first report of misdiagnosed PE due to aberrant aortopulmonary arterial connections. These striking sequences serve as a crucial reminder that interpretation of imaging in congenital heart disease is complex and should be considered carefully. The findings are instructive for all contrast-based imaging modalities in Tetralogy of Fallot and across the spectrum of congenital heart syndromes, where right-sided low-flow states predominate.
Contributors CMW: guarantor, history gathering, drafting the text, image acquisition and submission process. CJM: clinical management of case, conception of the work, critical revision of the article and final approval. PPP: critical revision of the article. CAR: management of case, conception of the work, critical revision of the article, image acquisition and final approval.

Funding The authors have not declared a specific grant for this research from any funding agency in the public, commercial or not-for-profit sectors.

Competing interests None declared.

Patient consent Obtained.

Provenance and peer review Not commissioned; externally peer reviewed.

\section{REFERENCES}

1 Sheikh AM, Kazmi U, Syed NH. Variations of pulmonary arteries and other associated defects in Tetralogy of Fallot. Springerplus 2014;3:467.

2 Nørgaard MA, Alphonso N, Cochrane AD, et al. Major aorto-pulmonary collateral arteries of patients with pulmonary atresia and ventricular septal defect are dilated bronchial arteries. Eur J Cardiothorac Surg 2006;29:653-8. 